\title{
Multi-user Driven Path Prediction Algorithm for Mobile Computing
}

\author{
M.Kyriakakos, S.Hadjiefthymiades, N.Frangiadakis, L.Merakos \\ Communication Networks Laboratory, \\ Department of Informatics and Telecommunications, \\ University of Athens, Panepistimioupolis, Athens 15784, Greece \\ \{miltos|shadj|ntg|merakos\}@di.uoa.gr
}

\begin{abstract}
Nowadays, path prediction is being extensively examined for use in the context of mobile and wireless computing towards more efficient network resource management schemes. Path prediction allows the network and services to further enhance the quality of service levels that the user enjoys. In this paper we present a path prediction algorithm that exploits the machine learning algorithm of learning automata. The decision of the learning automaton is driver by the movement patterns of a single user but is also affected by the aggregated patterns demonstrated by all users. Simulations of the algorithm, performed using a Realistic Mobility Pattern Generator, show increased prediction accuracy.
\end{abstract}

\section{INTRODUCTION}

The occurrence of handovers in cellular mobile networks is a very important issue and constitutes the main research drive for the design of resource management algorithms in such infrastructures. Network engineers are mostly concerned with the elimination of the problems induced by the disruptions in physical connectivity \& service provision. During the recent past, the pro-active method involving movement (path) prediction has been adopted for overcoming such problems in many diverse aspects of mobile computing. Pro-active management of network resources, driven by a properly structured movement estimator, enables the network designer to bypass (instead of correcting) the negative side effects of handovers. Network resources that may be administered through this scheme include (but are not limited to) bandwidth, MAC frames, files and packets. The network mechanisms, acting before the occurrence of the handover, reserve bandwidth or MAC frames, relocate files or packets to the best candidate (most likely) of the current cell's neighborhood. After the occurrence of the handover, the mobile terminal does not compete for such finite network resources (i.e., the bandwidth capacity in the new base station is limited; the same applies to file storage area or buffer capacity) but enjoys a pre-arranged situation. Hence, the nomadic user does not experience discontinuations (increased drop probability) or low performance in the provision of the service (i.e., a voice call).

In this paper, we present a variant of a path prediction algorithm initially presented in [5]. The considered algorithm is based on a technique taken from Artificial Intelligence (AI) namely a Learning Automaton (LA) [7]. The learning automaton, based on a properly structured knowledge, assigns probability values to the neighborhood of the currently visited cell. The highest probability denotes the most likely to be visited cell. In our initial design, the algorithm's decisions were only affected by the history of movements of a single user. In this paper, we introduce a slightly modified scheme. Specifically, we exploit not only the per-user recorded information, but also a global learning automaton influenced by the movements of all the users monitored by the system. Our simulations show the benefit obtained through this modified treatment of the underlying knowledge. Our simulations were realized on a mobility pattern simulator platform, called RMPG.

The rest of the paper is structured as follows. In Section 2, we present relevant prior work. In the same section, we also elaborate on the details of the learning automatonbased prediction algorithm. A variant of the original algorithm is presented. In Section 3, we briefly discuss the details of our mobility pattern simulator (RMPG). We use this simulator to evaluate the different variants of the prediction algorithm. Our results are presented to the reader in Section 4. Final conclusions and directions for further work in the area are included in Section 5.

\section{PATH PREDICTION ALGORITHMS}

In this section, after going through prior work on the same issue, we discuss the design of the LA-based prediction algorithm, both for the single-user and multi-user driven alternatives. The basis of our work in the design discussed in [5]. 


\subsection{Prior work}

In this section we briefly present other research efforts on the issue of predicting the movement of a nomadic user and pro-actively performing resource management.

In [1], the authors use the history of handoffs to predict the next cell. The information of handoffs is stored in a profile server, which translates the handoff histories into mobility profiles. These mobility profiles represent the mobility pattern of each user. At each base station, and for every user, a next-cell prediction algorithm is performed which informs the predicted neighbor cell for advance resource reservation. Two steps are followed. In the first step, when a user hands off from cell $c^{\prime}$ to cell $c$, the user profile is used to predict the next-cell. In the case of a success the probability $\mathrm{p}_{\mathrm{c}^{\prime}, \mathrm{c}^{\prime \prime}}$ for each neighbor cell $\mathrm{c}^{\prime \prime}$ of $\mathrm{c}$ is calculated for the prediction of the next cell. Otherwise the second step is followed. In the second step, the cell profile is used and similar steps are performed. Additional processing is taking place depending on the type of the cell.

A probabilistically model of the history of handoff behavior is proposed in [3]. The model is based in the aggregate history of all hand-offs that occurred in a cell. There are two stages, the handoff estimation and the predictiveadaptive bandwidth reservation. In the first stage, each BS keeps a database, which cashes a terminal's quadruplet ( $\mathrm{T}_{\text {event }}$, prev, next, $\mathrm{T}_{\text {soj }}$ ) called "a hand-off event quadruplet". $\mathrm{T}_{\text {event }}$ is the time when a terminal departed from the current cell, prev is the index of the previous visited cell, next is the index of the next cell, $\mathrm{T}_{\text {soj }}$ is the sojourn (residence) time of the terminal in the current cell. Through the cached quadruplets, each base station builds a function, called "hand-off estimation function", which outlines the allocation of the next cell and the sojourn time of a terminal.

The algorithm proposed in [2] is based on the LempelZiv algorithm and is called "LeZi-update". The idea is to reduce the update cost of the already existing update mechanism. The update scheme is a combination of time and movement based schemes. Every time the mobile user is crossing the boundaries of a cell, a record of the cell-id is kept in the cache. If the mobile user has visited in the past a cell it does not keep a record when revisiting it until the threshold of the movement-based scheme has been reached. The LeZi-update algorithm lies on the above update scheme and uses the movement history obtained from it.

The algorithm discussed in [6] is based on Mobile Motion Prediction (MMP) scheme for the prediction of the future location of a roaming user according to his movement history patterns. The scheme consists of RegularityPattern Detection (RPD) algorithms and Motion Prediction Algorithm (MPA). Regularity Detection is used to detect specific patterns of user movement from a properly structured database (IPB: Itinerary Pattern Base). Three classes of matching schemes are used for the detection of patterns namely the state matching, the velocity or time-matching and the frequency matching. The Prediction Algorithm (MPA) is invoked for combining regularity information with stochastic information (and constitutional constraints) and thus, reach a decision - prediction for the future location (or locations) of the terminal. Simulations of the proposed scheme have shown a maximum prediction efficiency of $95 \%$.

\subsection{Path Prediction using Learning Automaton}

In this paper, we propose a variant of an existing algorithm [5], which is based on a well-established AI technique for machine learning. More specifically, we build upon a learning automaton [7].

Learning automata are finite state adaptive systems that interact continuously in an iterative fashion with the environment. Through a probabilistic, trial-and-error response process they learn to choose or adapt to a behavior which generates the best response. Initially, an input is provided to the automaton from the environment. This input triggers one of a finite number of candidate responses from the automaton. The environment receives and evaluates the response and then provides feedback to the automaton. Such feedback is used by the automaton to alter its stimulus-response mapping structure to improve its behavior.

Learning automata are generally considered as robust but not very efficient learners. They are relatively easy to implement. Generally, the operation of the learning automaton is based on a state transition matrix, which contains the one-step transition probabilities $\mathrm{P}_{\mathrm{ij}}$ from the current state $\mathrm{i}$ to the next state $\mathrm{j}$. Different approaches have been proposed for the updating of the state transition matrix after the reception of environment feedback. In this paper we adopt the behavior of a Linear Reward-Penalty ( $\left.\mathrm{L}_{\mathrm{R}-\mathrm{P}}\right)$ Scheme. When the automaton selects the right response, the positive feedback received by the environment causes the respective state transition to be "rewarded" (i.e., its probability is increased by some pre-arranged step) while the probabilities of the state transitions that were not selected (remaining transitions from the same state) are "penalized" (decreased) uniformly to keep the probability sum to 1. If the proposed response is not appropriate (i.e., a negative feedback was received from environment) a reverse approach is followed; the probability value of the selected transition is "penalized", while the remaining transitions are evenly rewarded to balance the decrease. This behavior is shown in (Eq. 1):

$$
\begin{aligned}
& \text { Transition }(\mathrm{i} \rightarrow \mathrm{j}) \text { received positive feedback }:\left\{\begin{array}{l}
P_{i j}=P_{i j}+w\left(1-P_{i j}\right) \\
P_{i k}=P_{i k} \cdot(1-w), k \neq j
\end{array}\right. \\
& \text { Transition }(\mathrm{i} \rightarrow \mathrm{j}) \text { received negative feedback }:\left\{\begin{array}{l}
P_{i j}=P_{i j}-w^{\prime}\left(1-P_{i j}\right) \\
P_{i k}=P_{i k} \cdot\left(1+w^{\prime}\right), k \neq
\end{array}\right.
\end{aligned}
$$


In (Eq.1), w denotes the rewarding step while w'denotes the penalizing step. Those two values may be equal or different. In our implementation/simulation, both $\mathrm{w}$ and w'assumed different, yet constant values. Upon invocation, the automaton selects, as candidate future state, the state with the highest probability. After consecutive interactions with the environment, some state transitions will have probabilities close to 1 while others will have near-zero values (convergence). The structure of the originally proposed database is shown in Figure 1. This structured is extended with the "User_ID" field in order to host in the same database entries pertaining to more than one users.

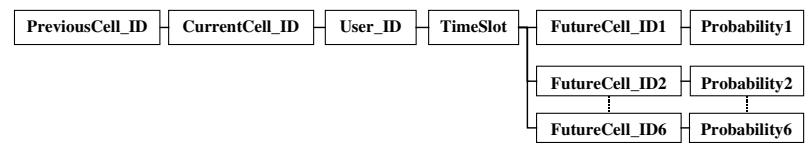

Figure 1. Layout of database for Per-User Automaton

In our model, time is assumed divided in slots of 15 minutes (e.g., only values like 10:00, 10:15, 10:30, 10:45 can be found). Whenever a prediction request arrives at the home registry of the mobile terminal the entry pertaining to the chronologically closest time slot is taken into account provided that the distance to it does not exceed the 1 hour limit (i.e., 4 time slots). If no appropriate state transitions were found then a new entry is fed into the database. If the automaton decision is correct (positive feedback) then the entry field that contributed to this decision is rewarded while the probability values of the remaining fields are reduced (penalized) as discussed.

When a mobile terminal powers-up in a new cell (not previously visited by the same terminal), a single record is automatically inserted in the itinerary database. This record contains fields with equal probability values (i.e., 1/6 for cells assumed hexagonal in shape), the current cell identification (CurrentCell_ID). The identification numbers of future (FutureCell_ID) and previous (PreviousCell_ID) cells reflect all the adjacent cells.

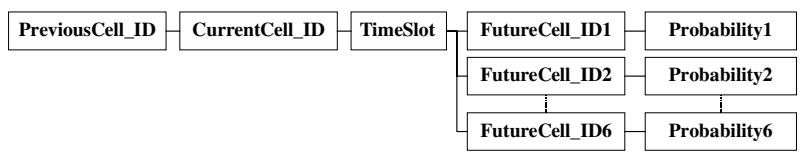

Figure 2. Layout of database for Global Automaton

The variant of the algorithm that we propose in this paper operates as follows. Two learning automata, the Global (GA) and the Per-User (PUA), operate simultaneously on top of two itinerary databases which have the layouts shown in Figure 2 and Figure 1 respectively, for some specific user. The set of entries in Figure 2, show the probabilities for specific cell crossings (i.e., Previous Cell $\rightarrow$ Current Cell $\rightarrow$ Future Cell), in a given time slot, for all users covered by the system. The reward/penalize proce- dures in this database are applied to the appropriate entries irrespective of the identifier of the user.

$W A($ previous, current, future, timeslot, uid $)=$

$a \cdot G A($ previous, current, future, timeslot $)+$

$(1-a) \cdot \operatorname{PUA}($ previous, current, future, timeslot, uid $)$,

$a=1 / 3$

When a prediction is requested, for a specific user, both automata (per-user and global) are consulted. The adopted scheme of combining the outcomes of both automata is termed Weighted Automaton (WA). The WA practically, combines the per-cell probabilities returned by the two automata using predefined weights. (Eq. 2) shows the calculation performed by WA. The reward/penalize procedure is applied independently to the PUA and the GA.

\section{REALISTIC MOBILITY PATTERN GENERATOR}

As discussed in Section 1, the performance assessment of the proposed path prediction algorithm has been based on a fully controllable mobility pattern generator platform developed in our laboratory. Such platform is called RMPG (Realistic Mobility Platform Generator). RMPG's design [4], is "based on the fact that everyone has some degree of regularity in his/her movement, that is, the movement of people consists of random movement and regular movement and the majority of mobile users has some regular daily (hourly, weekly, ....) movement patterns and follow these patterns more or less every day..."[6].

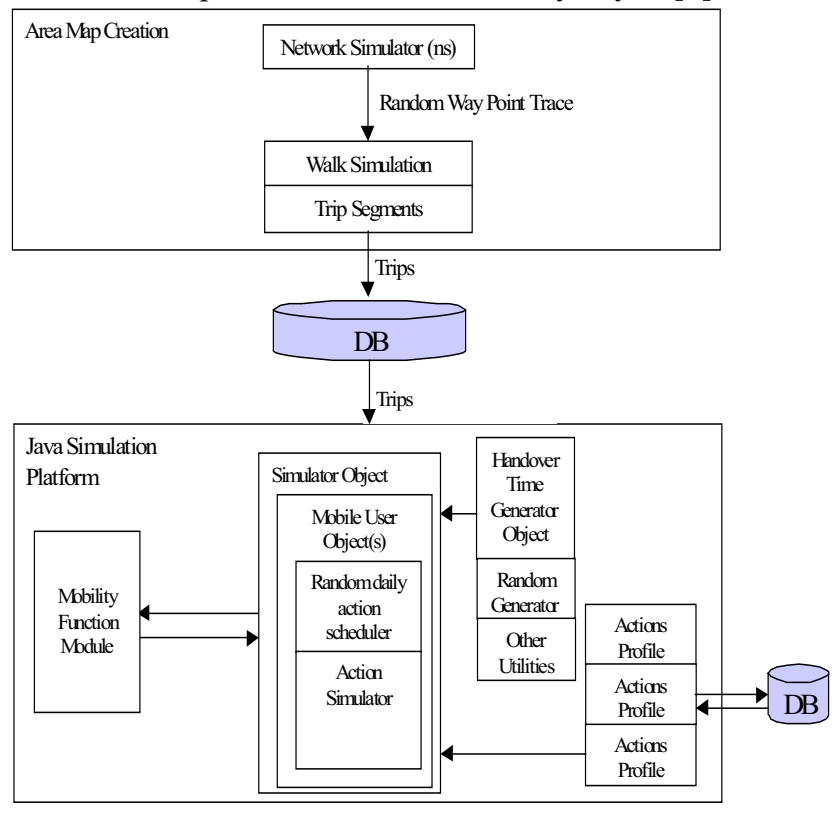

Figure 3. RMPG Architecture

RMPG can be used as a simulation platform for testing mobility functions like protocols, mobile architectures and applications under realistic workload conditions. In 
Figure 3 the overall architecture of RMPG is showed. Its operation is divided in two distinct stages (corresponding to the upper and the lower part of the figure respectively).

In the first stage the characteristics of the area, where mobile users roam, are defined. After completing the process titled "Area Map Creation", the allowed moves in the space are recorded using the Cell Ids (CIDs) of visited Base Stations (BS). The result is a set of Trips (fixed length sequences of CIDs) describing a "mobility map" of the area. In the second stage, the movement of users along the area is simulated. Each user may have a different role profile (businessman, student, etc.) and, therefore, exhibit a quite different behavior from others. The movement of users is constantly fed to the Mobility Function module allowing various tests on different kinds of mobility algorithms to be performed.

A very important aspect in RMPG is the time taken to cross each cell in the identified path (i.e., cell residence time). Such time is provided by the Handover Time Generator Object, which, in turn, implements the Generalized Gamma Distribution (GGD). The GGD is considered the best fit for cell residence times [9]. Hence, even for the same path the information provided to the Mobility Function Object is always different. Each time the mobile user moves from one cell to another, the Mobility Function Object is notified and all needed information including current time, previous cell history, current cell and next cell is passed to it.

\section{Simulation Randomness Metrics}

When studying the performance of mobility, one basic question is how random the user movement really was? Did it really put the assessed algorithm into stress? We have defined two metrics that provide indications of the randomness of the generated pattern/simulation. In RMPG, each pattern is generated by simulating a user of a specified profile in a given area, hence two factors affect the randomness of our models:

- The general layout of the area where the movement takes place

- The movement profile of the user

The layout of the area where the movement takes place can also provide the means to determine the upper limit of randomness in any simulated profile. As most stochastic pattern we consider that of a purely stochastic walk throughout the considered area. In an area full of obstacles, even such a walk may not be as random as we anticipated. Let $\rho_{\text {space }}$ denote the randomness of the space where the simulation took place. We define $\rho_{\text {space }}$ as follows:

$$
\rho_{\text {space }}=\frac{\sum_{i=1 . . n} F_{i}}{6 \cdot n}, 0 \leq \rho_{\text {space }} \leq 1
$$

In (Eq. 3), $\mathrm{n}$ denotes the total number of cells in the simulation terrain. $F_{i}$ denotes the number of feasible transitions from cell $i$ to adjacent cells $(6 \geq i \geq 1)$. For obstaclefree space, $F_{i}=6 \forall i$ and $\rho_{\text {space }}=1$. The profile of the user is very significant to determine how stochastic a derived pattern would be. In the extreme case that the profile determines a single itinerary for the user, his movement would be purely deterministic, while if he walks at random, the maximum degree of randomness in his mobility pattern is observed. We define $\rho_{\text {profile }}$ the user profile randomness. pprofile is calculated as follows:

$$
\begin{aligned}
& \rho_{\text {profile }}=\frac{T_{R W}}{T_{\text {total }}}+\frac{T_{\text {total }}-T_{R W}}{T_{\text {total }}} \cdot\left(1-\frac{A_{\text {active }}}{A_{\text {total }}}\right), \\
& 0<\rho_{\text {profile }} \leq 1
\end{aligned}
$$

In (Eq. 4), $\mathrm{T}_{\mathrm{RW}}$ denotes the time spent during the simulation in purely stochastic (random walk) actions while $T_{\text {total }}$ denotes the total simulation time. $A_{\text {total }}$ is the number of non-purely stochastic actions defined in the user's profile while $\mathrm{A}_{\text {active }}$ is the number of these actions that were executed in the course of the simulation. When the user is constantly involved in random walks, the $\rho_{\text {profile }}$ metric assumes a value of 1 . Should the fraction $A_{\text {active }} / A_{\text {total }}$ assume a value close to 1 (i.e., all the actions are executed with probability 1), the contribution of the second term in (Eq. 4) is negligible. In the case where the fraction $A_{a c-}$ tive $/ A_{\text {total }}$ assumes a value close to 0 (i.e., practically the execution of pre-defined actions is stochastic due to their high number), the second term of (Eq. 4) contributes to the $\rho_{\text {profile }}$ metric similarly to random walks.

\section{SIMULATION RESULTS}

We have programmed the path prediction algorithm in the Java-Language, a direction enforced by the operating platform of RMPG. We have logged the behavior of the algorithm throughout a period of 12 weeks. The rewarding step, $\mathrm{w}$ [5], assumed a random value of 0.1 while the penalizing step, $\mathrm{w}^{\prime}$, assumed a random value of 0.02 . The algorithm was applied to the movement of 10 mobile users within a predefined rectangular area. In RMPG's "Area Map Creation" the random way-point algorithm has been exploited. Specifically, the Network Simulator 2 (NS2) [8] has been used in order to obtain a random way-point trace. The users are moving in Trips of Trip size (number of CIDs contained in Trips) equal to 4 [4]. This, practically, denotes a relatively free of obstacles environment.

In the results presented below, the space randomness assumes the value $\rho_{\text {space }}=0.75$. In terms of the randomness of the user's profile, three profiles were considered. As expected, simulations have shown that for an increased value of $\rho_{\text {profile }}$ the accuracy of the automaton drops. Otherwise, low values of $\rho_{\text {profile }}$ cause highly accurate predictions. Hence, the reported accuracy climbs to $70-80 \%$ (which 
appears to be the ceiling in the performance achieved by the prediction algorithm).

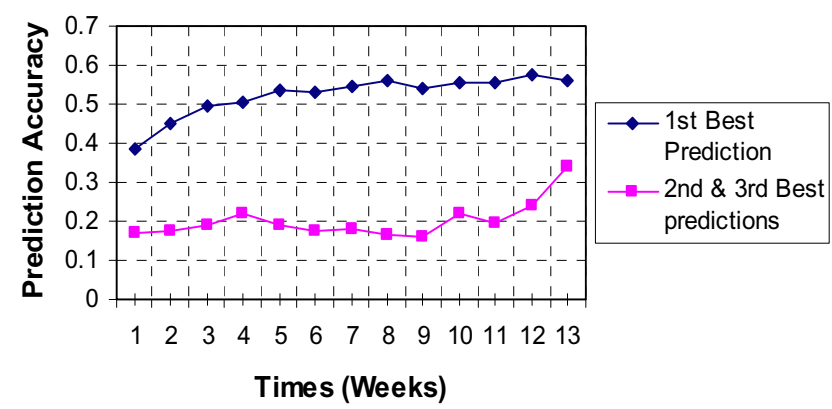

Figure 4. GA-Simulation Results

Figure 4 plots the performance achieved by the GA. The most probable cell to be visited by the roaming user comprises the best prediction of the automaton (shown as " 1 st Best Prediction"). In the same way, the second and third best predictions are examined. In Figure 4, apart from the best prediction accuracy we have also included the cumulative accuracy rate of the second and third best prediction. We can see that the maximum of the best prediction verges on $60 \%$, a behavior that is considered quite good for the prediction algorithm. Simulation results for the PUA are reported below.

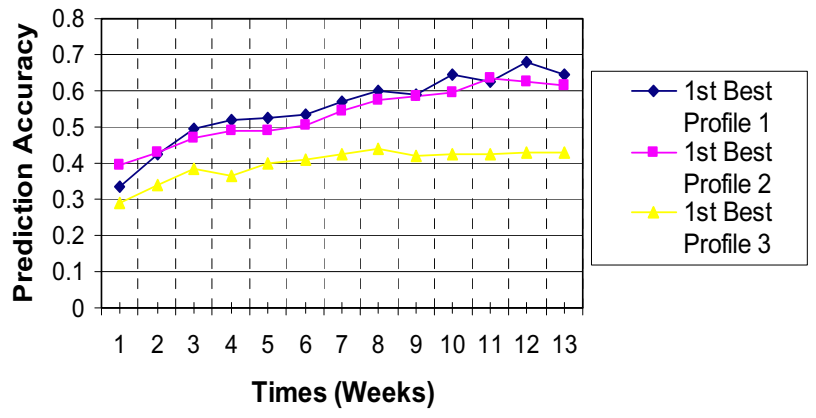

Figure 5. PUA-1st Best Prediction

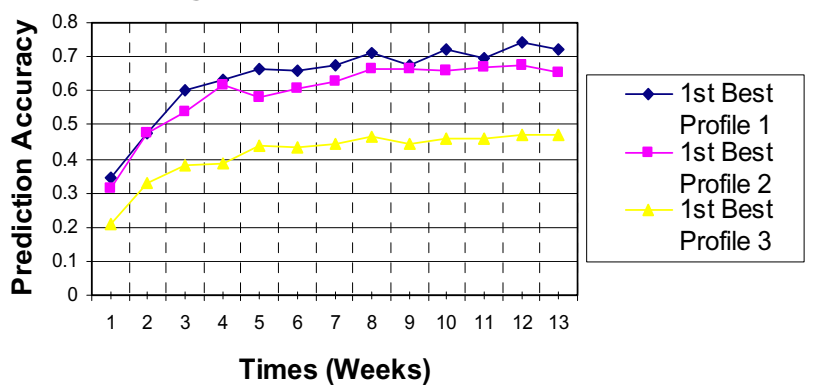

Figure 6. WA-1st Best Prediction

In Figure 5, we have plotted the average of the 1st Best Prediction accuracy for each simulated profile type. The numbers of the users on each simulated profile are 3 for Profile A, 3 for Profile B and 4 for Profile C (total: 10 users). It can been observed that, compared to the GA results, PUA results are worst in the beginning and get better in the concluding period of the simulation. In Figure 6 we present the simulation results for the WA also as an average of the 1 st best prediction for each simulated profile. It can be seen that the accuracy achieved by WA surpasses both the performance of GA and that of the PUA. The reported results show that even from the initial weeks, a higher prediction rate is succeeded against the PUA. Such behavior is maintained until the end of the simulation.

\section{CONCLUSIONS}

In this paper, we have discussed the design and performance evaluation of a path prediction scheme based on the learning automaton algorithm for machine learning. We have presented an enhanced algorithm that takes into account not only the past behavior of a specific user but also the aggregated behavior of all other users controlled by the same system. The algorithm has been simulated through a realistic mobility pattern generator. Simulation results show that the system achieves high prediction accuracy when the combined decision scheme is adopted. Prediction accuracy in the order of $70-80 \%$ seems very promising for mobile/wireless computing solutions and architectures. Currently, we are extending our learning automaton work so that different values of the reward/penalize step are evaluated but also a more dynamic scheme where such parameter is adjusted in an ad hoc way.

\section{REFERENCES}

[1] V.Bharghavan, and M. Jayanth, "Profile-based Next-cell Prediction in Indoor Wireless LAN," in proceeding of IEEE SICON'97, Apr. 1997.

[2] A. Bhattacharya, and S. K. Das, "LeZi Update: An Information Theoretic Approach to Track Mobile Users In PCS Networks", proceedings of ACM/IEEE Mobicom '99, Seattle, USA, August 1999.

[3] S., Choi, and K.G., Shin, "Predictive and Adaptive Bandwidth Reservation for Hand-offs in QoS-Sensitive Cellular Networks", proceedings of ACM SIGCOMM '98, Vancouver, British Columbia, September 1998.

[4] N. Frangiadakis, M. Kyriakakos, S. Hadjiefthymiades, L. Merakos "Realistic Mobility Pattern Generator: Design and Application in Path Prediction Algorithm Evaluation", proceedings of IEEE PIMRC conference, Lisbon, Portugal, 2002.

[5] S.Hadjiefthymiades, and L.Merakos, "ESW4: Enhanced Scheme for WWW computing in Wireless communication environments", ACM SIGCOMM Computer Communication Review, Vol.29, No.5, 1999.

[6] George y. Liu, Gerald Q. Maguire Jr. " A Class of Mobile Motion Prediction Algorithms for Wireless Mobile Computing and Communications “, MONET, Vol.1, pp.113-121, 1996.

[7] K. Narendra, and M. A. L. Thathachar, "Learning Automata: An Introduction", Prentice-Hall, 1989.

[8] http://www.isi.edu/nsnam/ns/

[9] M.Zonoozi, and P.Dassanayake, "User Mobility Modeling and Characterization of Mobility Patterns", IEEE Journal on Selected Areas in Communications, Vol.15, No.7, 1997. 